\title{
A knowledge audit model to assess the knowledge in requirement elicitation process
}

\begin{abstract}
There is a great deal of knowledge in requirement elicitation process (REP), because there are different stakeholders with various knowledge backgrounds. Different backgrounds of knowledge lead to different ways of knowledge expression that negatively affect knowledge understandability and cause ambiguity. Knowledge ambiguity results in incorrect interpretation of knowledge and requirements. On the other hand, different stakeholders have different needs and expectations from the software to be developed. This problem causes conflicting information and also negatively affects the correctness of knowledge. Furthermore, stakeholders may ignore mentioning some knowledge because they think it is obvious or their requirements change over time, this negatively affects completeness of knowledge in REP. To mitigate these problems, it is necessary to identify and assess the knowledge in REP. Knowledge Audit (KA) is the process of knowledge analysis and assessment. Therefore, this research introduces a KA model to support knowledge communication among stakeholders through objectively assessing the knowledge in REP.
\end{abstract}

Keyword: Knowledge assessment; Knowledge audit; Knowledge in requirement elicitation process; Requirement elicitation process 